\title{
Machine learning modelling for predicting soil liquefaction susceptibility
}

\author{
P. Samui ${ }^{1}$ and T. G. Sitharam ${ }^{2}$ \\ ${ }^{1}$ Centre for Disaster Mitigation and Management, VIT University, Vellore - 632014, India \\ ${ }^{2}$ Department of Civil Engineering, Indian Institute of Science, Bangalore - 560012, India
}

Received: 14 December 2009 - Revised: 20 September 2010 - Accepted: 21 September 2010 - Published: 3 January 2011

\begin{abstract}
This study describes two machine learning techniques applied to predict liquefaction susceptibility of soil based on the standard penetration test (SPT) data from the 1999 Chi-Chi, Taiwan earthquake. The first machine learning technique which uses Artificial Neural Network (ANN) based on multi-layer perceptions (MLP) that are trained with Levenberg-Marquardt backpropagation algorithm. The second machine learning technique uses the Support Vector machine (SVM) that is firmly based on the theory of statistical learning theory, uses classification technique. ANN and SVM have been developed to predict liquefaction susceptibility using corrected SPT $\left[\left(N_{1}\right)_{60}\right]$ and cyclic stress ratio (CSR). Further, an attempt has been made to simplify the models, requiring only the two parameters $\left[\left(N_{1}\right)_{60}\right.$ and peck ground acceleration $\left.\left(a_{\max } / \mathrm{g}\right)\right]$, for the prediction of liquefaction susceptibility. The developed ANN and SVM models have also been applied to different case histories available globally. The paper also highlights the capability of the SVM over the ANN models.
\end{abstract}

\section{Introduction}

Liquefaction is a phenomenon whereby a granular material transforms from a solid state to a liquefied state consequently of the increase in pore water pressure. The effective stress of the soil reduces, therefore, causing loss of bearing capacity. There are three types of damage occurring during liquefaction. First is that ground lateral spreading and failures of dam embankment are the particular types of landslides which could be classified as liquefaction (Keefer, 1984). Second is that sand blows and ground cracks are the surface manifestations of liquefaction in

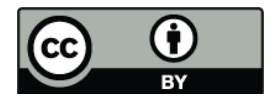

Correspondence to: P. Samui

(pijush.phd@gmail.com) soil. Third is that building settlement and/or severe tilting are the hazardous consequences of the liquefaction. The Damages attributed to the earthquake-induced liquefaction phenomenon have cost society hundreds of millions of US dollars (Seed and Idriss, 1982). Therefore, the assessment of the liquefaction potential due to an earthquake at a site is an imperative task in earthquake geotechnical engineering. The liquefaction susceptibility of soil depends on the earthquake parameter and soil parameter. One of the most important earthquake parameter is the maximum epicentral distance (Papadopoulos and Lefkopoulos, 1993). Kramer (1996) has described the different soil parameters such as fraction finer than $0.005 \mathrm{~mm}$, liquid limit, natural water content, liquidity index, gradation, particle shape, initial state of the soil, etc. A procedure based on standard penetration test (SPT) and cyclic stress ratio (CSR) was developed by Seed and his colleagues $(1967,1971,1983,1984)$ based on the use of peck ground acceleration ( $\mathrm{PGA}=a_{\max } / \mathrm{g}$ ) to assess the liquefaction potential of soil, and is now in standard use around the world. Liao et al. (1988) and Cetin (2000) used a probabilistic framework to model the variability and uncertainty inherent to the problem of liquefaction. Goh (1994) successfully applied Artificial Neural Network (ANN) for the determination of liquefaction susceptibility of soil. However, ANN models have some limitations such as the black box approach, arriving at local minima, slow convergence speed and over fitting problems (Park and Rilett, 1999; Kecman, 2001).

In this paper, two machine learning techniques (ANN and support vector machine, SVM) have been adopted to predict liquefaction susceptibility of soil based on the standard penetration test (SPT) data from the 1999 Chi-Chi, Taiwan earthquake. The epicentre of the earthquake was at $23.87^{\circ} \mathrm{N}$, $120.75^{\circ} \mathrm{E}$ (Juang et al., 2002). This earthquake caused a lot of damage, in particular large scale liquefaction in Central Taiwan (Juang et al., 2002). Several SPT tests were conducted subsequent to the earthquakes in the different sites

Published by Copernicus Publications on behalf of the European Geosciences Union. 
(Mingjian Shiang, Taiping City, Wufeng Shinag, Yuanlin Jen, Taichung Harbour, Chiuanshing, Mingjian Shiang, Chanbing Industrial Park, Nantou City, etc.) and the results have been published (Hwang and Yang, 2001). The SPT test also gives the information about fines content, depth of water table, clay size content, $D_{50}$, SPT energy ratio, etc. Information (liquefaction, soil profile, etc) about ChiChi earthquake was given by different researchers (Lin et al., 2001; Lee et al., 2001, 2001; Wang et al., 2002, 2003, 2004; Sokolov et al., 2002; Shou and Wang, 2003; Yuan et al., 2004; Chu et al., 2004; Ku et al., 2004; Chua et al., 2004). ANN has been used with multilayer perceptrons (MLPs) that are trained with LevenbergMarquardt backpropagation algorithm. The Support Vector Machine (SVM) based on statistical learning theory has been developed by Vapnik (1995). This study employs SVM as a classification technique. Two sets of analyses were carried out - first by using the two input parameters, corrected SPT $\left[\left(N_{1}\right)_{60}\right]$ and CSR and the second one using $\left(N_{1}\right)_{60}$ and $\mathrm{a}_{\max } / \mathrm{g}$. The developed models have been tested for different case histories available globally (Goh, 1994). A comparative study has also been carried out between the developed ANN and SVM models.

\section{Methodology}

In this paper, two models (ANN and SVM) have been adopted for prediction liquefaction susceptibility. Brief descriptions of the two models developed for our study are given below.

\subsection{ANN model}

In this study, MLPs that are trained with LevenbergMarquardt Backpropagation algorithm has been used (Hagan and Menhaj, 1994). MLPs are perhaps the best-known type of feed forward networks. It has generally three layers: an input layer, an output layer and an intermediate or hidden layer. In the backpropagation training process, the network error is back propagated into each neuron in the hidden layer, and then continued into the neuron in the input layer. The modification of the connection weights and biases depend on the distribution of error at each neuron. The global network error is reduced by continuous modifications of connection weights and biases. An error goal is set before the network training, and if the network error during the training becomes less than the error goal, the training has to be stopped. Levenberg-Marquardt backpropagation algorithm is a variation of Newton's method and is well-suited to ANN training. The theory and implementation of LevenbergMarquardt Backpropagation has been given by More (1977).

The main scope of this study is to implement the ANN backpropgation methodology in the prediction of liquefaction susceptibility based on the actual SPT field data from Chi-Chi, Taiwan earthquake by developing two models (MODEL I and MODEL II). This study uses the database collected by Hwang and Yang (2001). Out of the total 288 datasets, a total of 164 data are for the sites which are liquefied and 124 are for non-liquefied sites after the earthquake. The liquefaction susceptibility of a soil mass during an earthquake is dependent on both seismic and soil parameters. So, in MODEL I, the input parameters are the corrected SPT value $\left[\left(N_{1}\right)_{60}\right]$ and cyclic shear stress ratio (CSR). To use these data for classification purpose, a value of -1 is assigned to the liquefied sites while a value of 1 is assigned to the non-liquefied sites so as to make this a two-class classification problem. So, the output of the model will be either 1 or -1 . The data is normalized against their maximum values (Sincero, 2003). In carrying out the formulation, the data has been divided into two sub-sets: such as

- A training dataset: this is required to construct the model. In this study, 202 data out of the 288 are considered for the training dataset.

- A testing dataset: this is required to estimate the model performance. In this study, the remaining 86 data are considered as testing dataset.

Researchers have used a different percentage of the available data as the training set for different problems. For instance, Kurup and Dudani (2002) used $63 \%$ of the data for training; Tang et al. (2005) used 75\%; while Padmini et al. (2008) used $80 \%$. In this study, we have used $70 \%$ of the data for training. The statistical consistency of training and testing datasets improves the performance of the ANN model and later helps in evaluating them better (Shahin et al., 2000).

CSR has been used as an input parameter in MODEL I. CSR has been calculated from the following formula (Seed and Idriss, 1971),

$\mathrm{CSR}=0.65\left(\frac{\sigma_{\mathrm{v}}}{\sigma_{\mathrm{v}}^{\prime}}\right)\left(\frac{a_{\max }}{g}\right)\left(\gamma_{\mathrm{d}}\right) / \mathrm{MSF}$

where, $\sigma_{\mathrm{v}}$ is total overburden stress, $\sigma_{\mathrm{v}}^{\prime}$ is effective overburden stress, $\gamma_{\mathrm{d}}$ is stress reduction factor and MSF is magnitude scaling factor. Because of the difficulty and cost constraint of obtaining high-quality undisturbed samples, it is very difficult to get a reliable value of $\sigma_{\mathrm{v}}$ and $\sigma_{\mathrm{v}}^{\prime}$. So, it is a very difficult task to determine CSR value accurately. The purpose of the development of MODEL II is to predict liquefaction based on $\left(N_{1}\right)_{60}$ and $a_{\max } / \mathrm{g}$. So in MODEL II, the input variables are $\left(N_{1}\right)_{60}$ and $a_{\text {max }} / g$. In MODEL II, the same training dataset, testing dataset and normalization technique have been used in MODEL I. MODEL II has also been verified for the additional 85 case histories (which were not part of training or testing dataset used earlier to develop the model) available globally (Goh, 1994). Both programmes (MODEL I and MODEL II) are constructed using a neural network tool box in MATLAB (Demuth and Beale, 1999). 


\subsection{SVM model}

SVM has originated from statistical learning theory pioneered by Boser et al. (1992). Since SVM is a relatively new technique, a brief explanation of how it works is given below. More details can be found in many publications (Boser et al., 1992; Cortes and Vapnik, 1995; Gualtieri et al., 1999; Vapnik, 1998). Consider the training datasets which consists of $k$ training samples represented by $\left(\boldsymbol{x}_{1}, y_{1}\right), \ldots,\left(x_{k}, y_{k}\right)$, where $\boldsymbol{x}_{i} \in R^{N}$ is an N-dimensional data vector with each sample belonging to either of the two classes labelled as $y_{i} \in$ $\{+1,-1\}$. In this study, for MODEL I $x=\left[\mathrm{CSR},\left(N_{1}\right)_{60}\right]$ and for MODEL II $x=\left[a_{\max } / g,\left(N_{1}\right)_{60}\right]$. To use the SPT data for classification purposes, a value of -1 is assigned to the liquefied sites while a value of 1 is assigned to the nonliquefied sites, so as to make this a two-class classification problem. The equation of a hyperplane that does the separation is

$\boldsymbol{w} \times \boldsymbol{x}+b=0, w \in R^{N}, \quad b \in R$

Where $\boldsymbol{x}$ is an input vector, $\boldsymbol{w}$ is an adjustable weight vector, $b$ is a bias, $R^{N}$ is N-dimensional real vector space and $R$ is one dimensional real vector space. For the linearly separable class, a separating hyperplane can be defined for the two classes as

$\boldsymbol{w} \times \boldsymbol{x}_{i}+b \geq+1$ for $y_{i}=+1 \rightarrow$ No liquefaction

$\boldsymbol{w} \times \boldsymbol{x}_{i}+b \leq-1$ for $y_{i}=-1 \rightarrow$ liquefaction

Sometimes, due to the noisy or mixture of classes of training data, variables $\xi_{i}>0$, called slack variable, are used to account for the effects of misclassification. So Eq. (3) can be written in the following way

$y_{i}\left(\boldsymbol{w} \times \boldsymbol{x}_{i}+b\right) \geq 1-\xi_{i}$

The optimal hyperplane is located where the margin between two classes is maximized and the error is minimized. The support vectors of the two classes lie on two hyperplanes, which are parallel to the optimal hyperplane and are defined by $\boldsymbol{w} \cdot \boldsymbol{x}_{i}+b= \pm 1$. The margin between these planes is $\frac{2}{\|\boldsymbol{w}\|}$. Maximization of this margin can be achieved by solving the following constrained optimization problem,

Minimize : $\frac{1}{2}\|\boldsymbol{w}\|^{2}+C \sum_{i=1}^{l} \xi_{i}$

Subjected to : $y_{i}\left(\boldsymbol{w} \times \boldsymbol{x}_{i}+b\right) \geq 1-\xi_{i}$

The constant (called capacity factor) $0<C<\infty$, a parameter defines the trade-off between the number of misclassification in the training data and the maximization of margin. This optimization problem (5) is solved by Lagrangian Multipliers (Vapnik, 1998). According to the Karush-Kuhn-Tucker (KKT) optimality condition (Fletcher, 1987), some of the multipliers will be zero. The nonzero multipliers are called support vectors (see Fig. 1). In conceptual terms, the

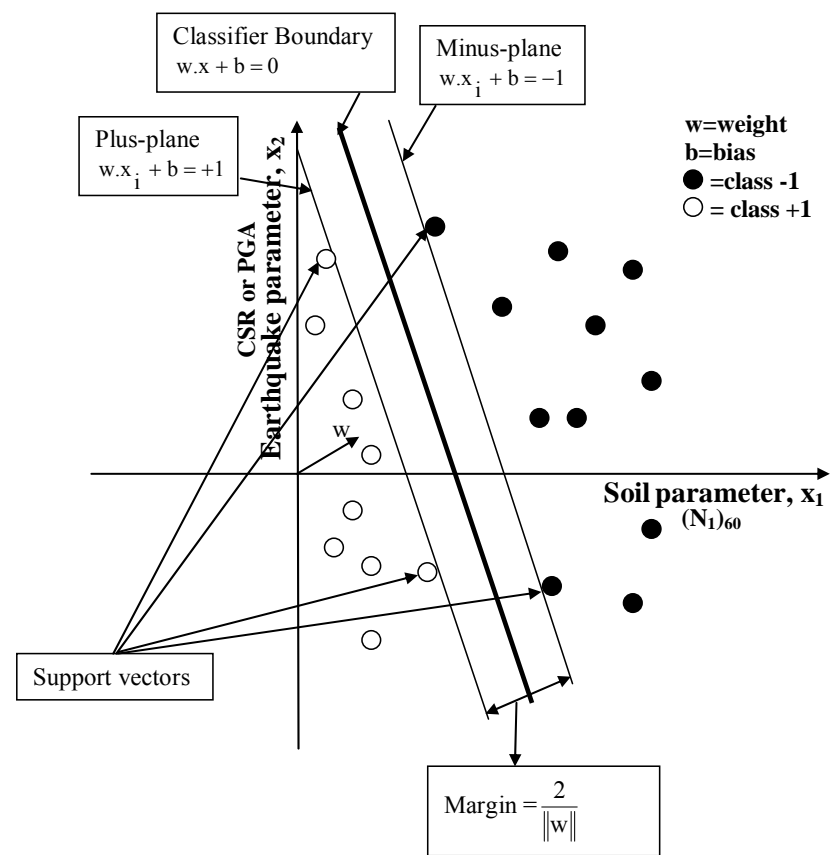

Fig. 1. Support vectors with maximum margin.

support vectors are those data points that lie closest to the optimal hyperplane and are, therefore, the most difficult to classify. The value of $\boldsymbol{w}$ and $b$ are calculated from $\boldsymbol{w}=$ $\sum_{i=1}^{l} y_{i} \alpha_{i} \boldsymbol{x}_{i}$ and $b=-\frac{1}{2} \boldsymbol{w}\left[x_{+1}+x_{-1}\right]$, where $x_{+1}$ and $x_{-1}$ are the support vectors of class labels +1 (No liquefaction) and -1 (liquefaction), respectively. The classifier can then be constructed as:

$f(x)=\operatorname{sign}(\boldsymbol{w} \times x+b)$

where sign is the signum function. It gives +1 (No liquefaction) if the element is greater than or equal to zero and -1 (liquefaction) if it is less than zero.

In case linear supporting hyper plane is inappropriate, the SVM maps input the data into a high dimensional feature space through some nonlinear mapping (Boser et al., 1992). This method easily converts a linear classification learning algorithm into a nonlinear one, by mapping the original observations into a higher-dimensional nonlinear space so that linear classification in the new space is equivalent to nonlinear classification in the original space. Kernel function has been introduced instead of feature space $(\Phi(x))$ to reduce computational demand (Cortes and Vapnik, 1995; Cristianini and Shwae-Taylor, 2000). To get the Eq. (6), same procedures have been applied as in the linear case.

Radial basis function has been used as kernel function in this study. In SVM, For MODEL I and MODEL II, the same training dataset, testing dataset, input variables and normalization technique have been used as in the ANN model. The application of SVM for this study requires the 


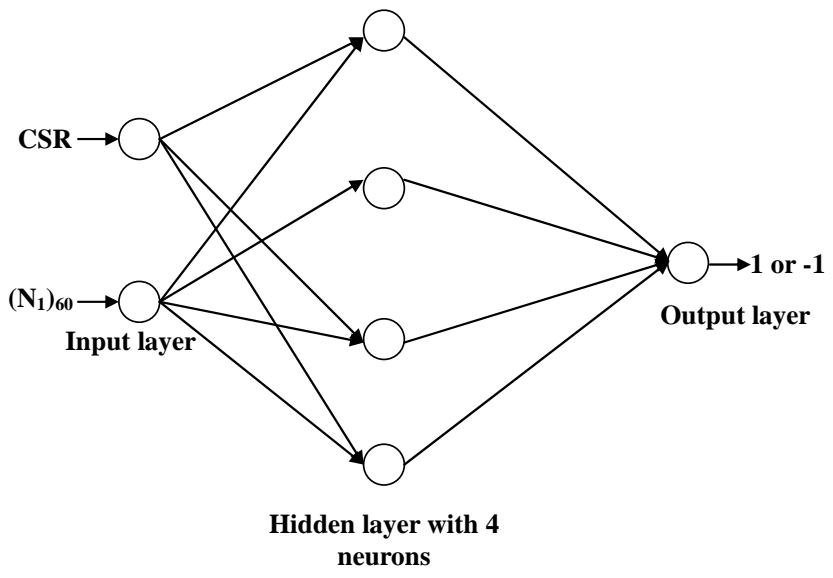

Fig. 2. ANN architecture for MODEL I.

proper selection of $\mathrm{C}$ value. The identification of optimal value of $C$ is largely a trial and error process. However, there are guidelines that can be used for selecting $C$. A large $C$ assigns higher penalties to errors so that the regression is trained to minimize error with lower generalization while a small $C$ assigns fewer penalties to errors; this allows the minimization of margin with errors, thus, a higher generalization ability. If $C$ goes to infinitely large, SVM would not allow the occurrence of any error and result in a complex model, whereas when $C$ goes to zero, the result would tolerate a large amount of errors and the model would be less complex.

\section{Results and discussion}

For predicting liquefaction susceptibility, the two input variables (CSR and $\left.\left(N_{1}\right)_{60}\right)$ are used for ANN model for MODEL I. Hence, the input layer has two neurons. The only output is the 1 or -1 and, therefore, the output layer has only one neuron. In ANN model, the optimum backpropagation networks that can be obtained in the present study are a three-layer feed forward network. Figure 2 shows the final architecture of the ANN model with one hidden layer. In this study, the transfer function used in the hidden layer is logsig. The expression of logsig is given below:

$\log \operatorname{sig}(x)=\frac{1}{1+\exp (-x)}$

The tansig transfer function has been used in the output layer. The expression of tansig is given below:

$\tan \operatorname{sig}(x)=\frac{2}{1+\exp (-2 x)}-1$

The number of neurons in the hidden layer is determined by training several networks with different numbers of hidden neurons and comparing the predicted results with the desired output. Using too few hidden neurons could result in huge

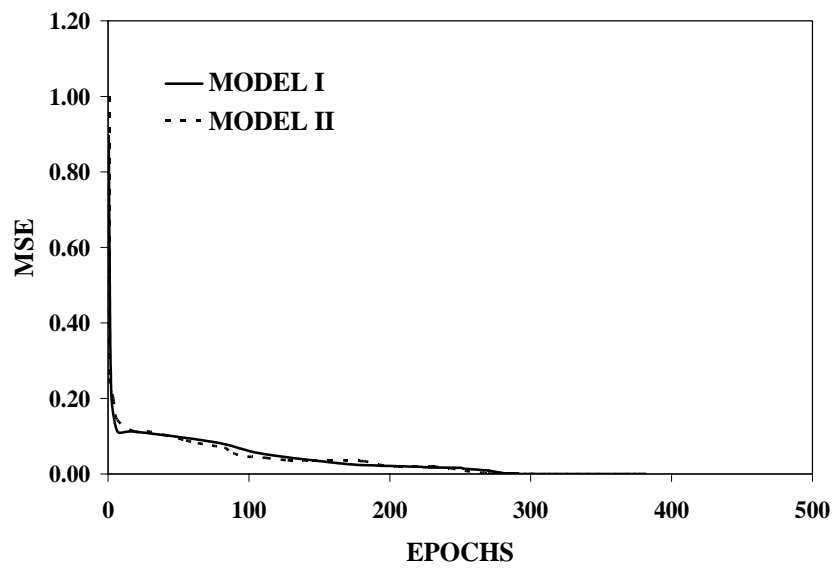

Fig. 3. MSE vs. Epochs for ANN models.

training errors and errors during testing, due to underfitting and high statistical bias. On the other hand, using too many hidden neurons might give low training errors but could still have high testing errors due to overfitting and high variance. In this study, the hidden layer with 4 neurons has been used. For ANN model, the converged results have been achieved at 382 epochs (an epoch is one complete presentation of the entire set of training patterns during the training process). The value of mean square error (MSE) for the ANN model has been computed and monitored during training. Figure 3 shows how the MSE for ANN model reduces as training proceeds. Training and testing performance $(\%)$ has been calculated by using the following formula:

Training performance $(\%)$ or Testing performance $(\%)$

$=\left(\frac{\text { No of data predicted accurately by ANN }}{\text { Total data }}\right) \cdot 100$

The performance of training data is $94.55 \%$. According to the results of network training, the network has successfully captured the relationship between the input parameters and output. In order to evaluate the capabilities of the ANN model, the model is validated with new data that are not part of the training dataset. In this case, the performance of ANN model is $88.37 \%$. Figures 4 and 5 illustrate the plot between CSR and $\left(N_{1}\right)_{60}$ for training and testing dataset, respectively. These figures provide a design assessment chart that can be used to estimate the liquefaction resistance of soils.

In MODEL II, the input variables are $a_{\mathrm{max}} / \mathrm{g}$ and $\left(N_{1}\right)_{60}$. So, the input layer has only two neurons. The output of the model is 1 or -1 . Hence, the output layer has only one neuron. MODEL II uses three layer feed forward network with 5 neurons in the hidden layer and it has been shown in Fig. 6. The variation of MSE with epochs has been shown in Fig. 3. For MODEL II, the converged results have been achieved at 295 epochs (see Fig. 3). The performance of training and testing dataset is 94.05 and 87.20 , respectively. So, there is a marginal reduction of 


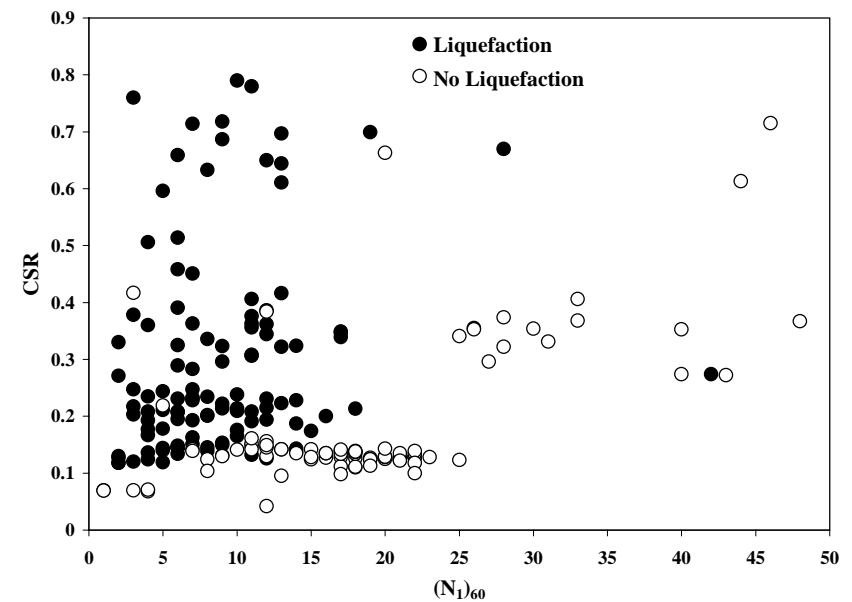

Fig. 4. Plot between CSR and $\left(N_{1}\right)_{60}$ for MODEL I using training dataset for ANN model.

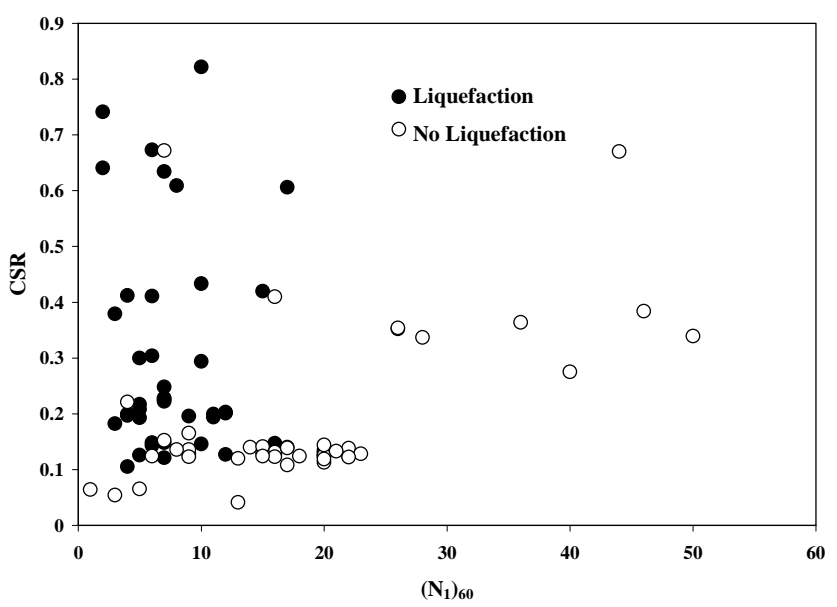

Fig. 5. Plot between CSR and $\left(N_{1}\right)_{60}$ for MODEL I using testing dataset for BP model.

performance of MODEL II compare to MODEL I. The value of $\left(N_{1}\right)_{60}$ represents the different properties of the soil. CSR is a function of different soil properties and earthquake parameters. Therefore, $\left(N_{1}\right)_{60}$ partly represent CSR value. For this reason, the performance of MODEL I and MODEL II are almost same. Figures 7 and 8 demonstrate the plot between PGA and $\left(N_{1}\right)_{60}$ for the training and testing dataset, respectively. The user can use these figures for separating liquefiable and non-liquefiable soil. This study indicates that the two input parameters [PGA and $\left.\left(N_{1}\right)_{60}\right]$ are sufficient to determine liquefaction susceptibility of the soil. There is no need to calculate the value of CSR. For global data, the performance of ANN model is $70.58 \%$. Figure 9 depicts the plot between PGA and $\left(N_{1}\right)_{60}$ for global data using the ANN model. The separation between liquefiable and non-liquefiable soil is quite the same for these three figures (7, 8 and 9).

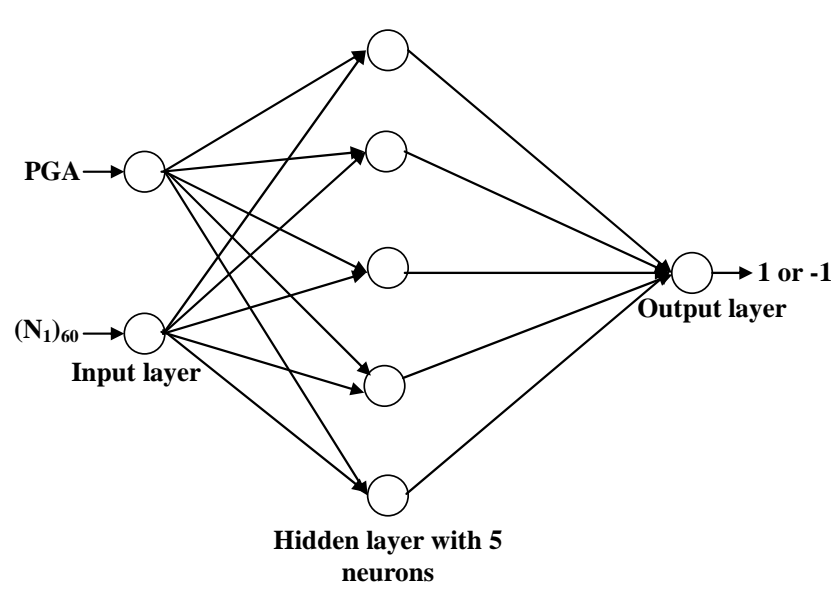

Fig. 6. ANN architecture for MODEL II.

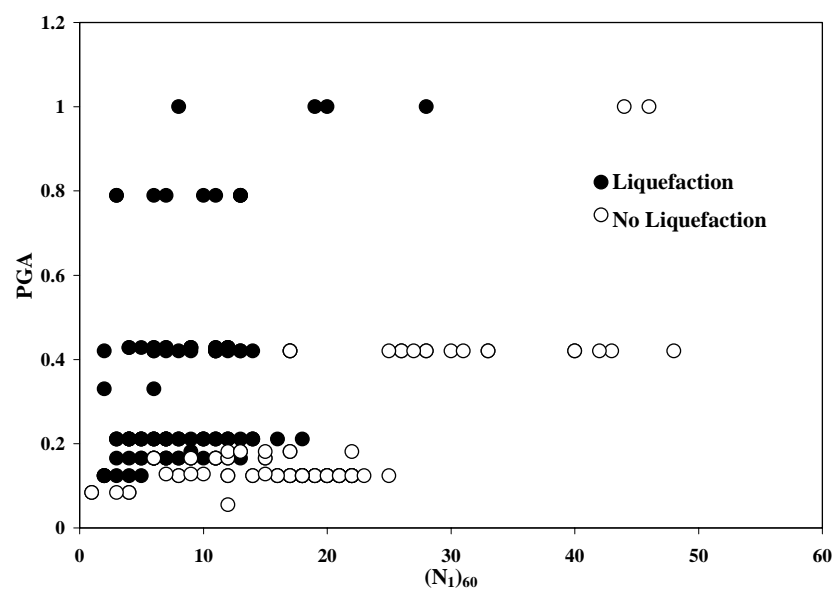

Fig. 7. Plot between PGA and $\left(N_{1}\right)_{60}$ for MODEL I using training dataset for ANN model.

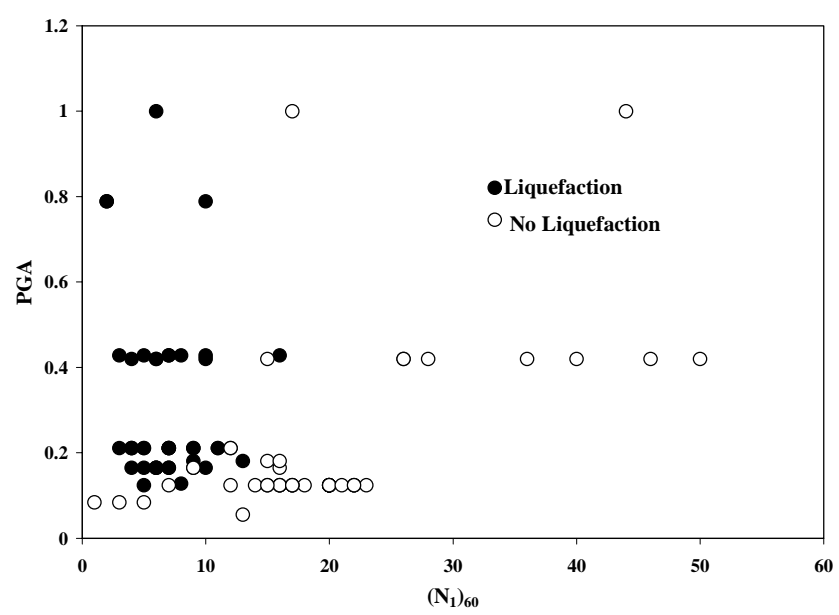

Fig. 8. Plot between PGA and $\left(N_{1}\right)_{60}$ for MODEL I using training dataset for ANN model. 


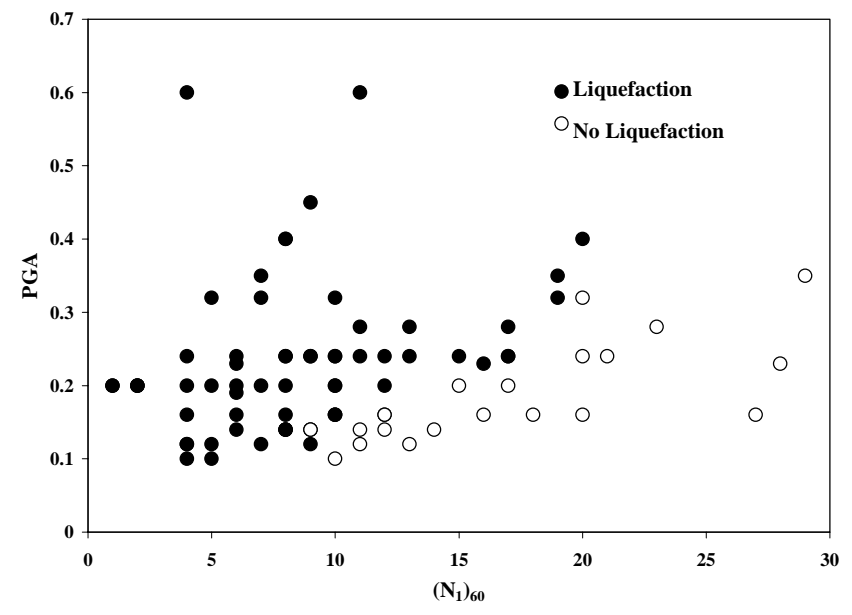

Fig. 9. Plot between PGA and $\left(N_{1}\right)_{60}$ for global data using ANN model.

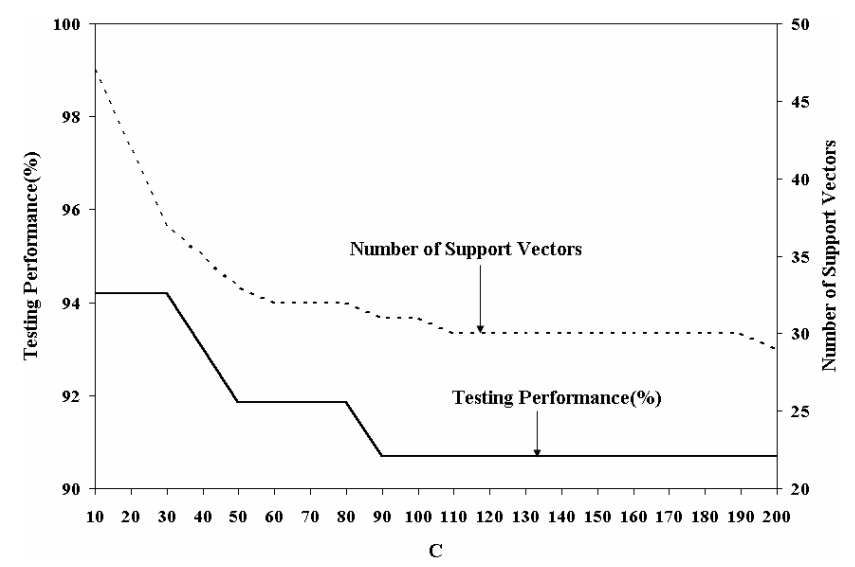

Fig. 10. Variation of Testing Performance (\%) and Number of Support Vectors with $\mathrm{C}$ values for MODEL I using radial basis function kernel.

Since there is no rule in selecting the C value of SVM, it is necessary to investigate the impact of $C$ on testing performance (\%) as well as the number of support vectors for each kernel. The training and testing performance (\%) of SVM has been determined by using the following 9 but for SVM. Figure 10 depicts the effect of $C$ on testing performance (\%) and the number of support vectors for MODEL I using radial basis function. Figure 10 demonstrates that the testing performance (\%) attains maximum value at $C=10$. Generally it can be seen, from Fig. 10, that the number of support vectors is decreasing when $C<120$ and tend to flatten after $C \geq 120$. For the best model, a high testing performance $(\%)$ as well as less support vectors is desirable. The design value of $C$ and width of radial basis function $(\sigma)$ is 30 and 0.4 , respectively. The number of support vector is 37 . Training and testing performance of MODEL I are $96.04 \%$ and

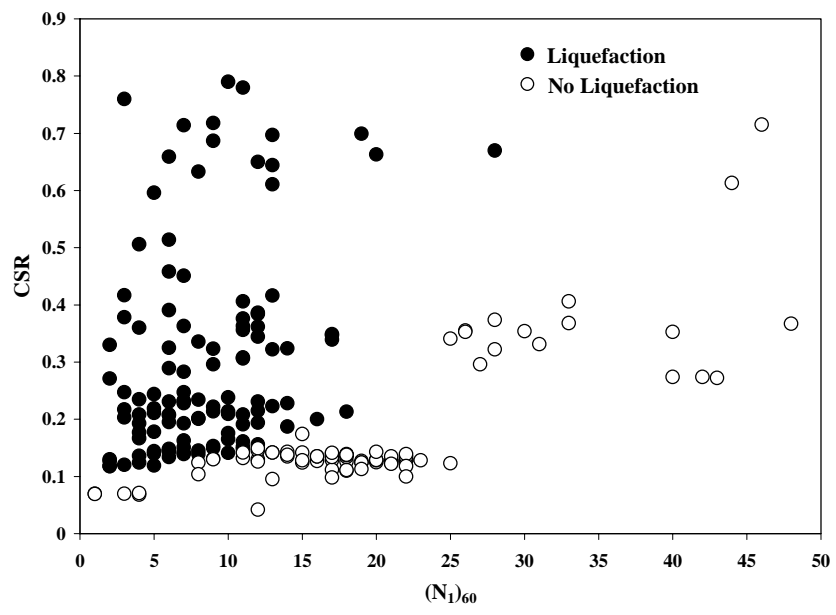

Fig. 11. Plot between CSR and $\left(N_{1}\right)_{60}$ for MODEL I using radial basis function for training dataset.

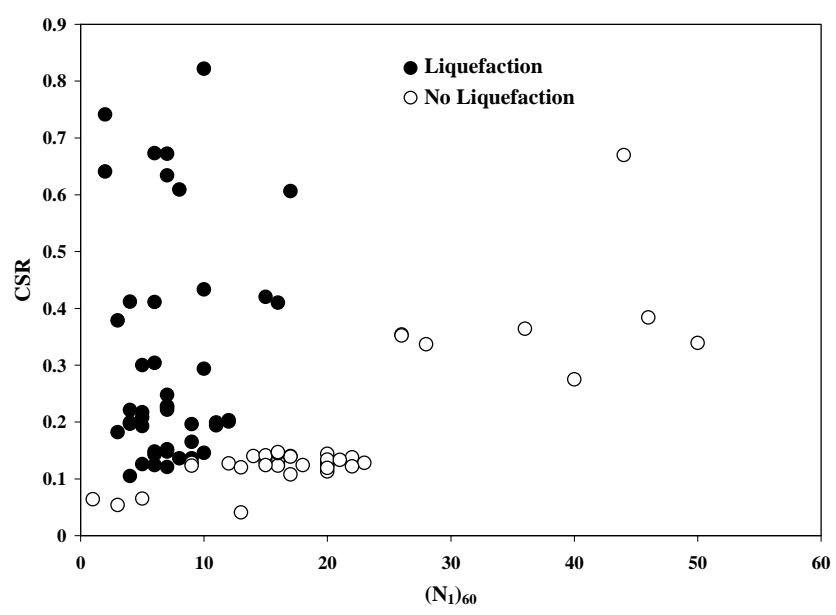

Fig. 12. Plot between CSR and $\left(N_{1}\right)_{60}$ for MODEL I using radial basis function for testing dataset.

94.19\%, respectively. Hence, this study indicates that SVM model has the ability to predict liquefaction susceptibility of soil based on SPT data. Figures 11 and 12 illustrate the plot between CSR and $\left(N_{1}\right)_{60}$ for training and testing dataset, respectively.

For MODEL II, Fig. 13 demonstrates the effect of $C$ on the testing performance $(\%)$ and the number of support vectors using radial basis function. It can be seen from Fig. 13 that testing performance (\%) is not affected by $\mathrm{C}$ value for radial basis function. For radial basis function kernel, $\mathrm{C}$ value does not affect the number of support vectors as observed in Fig. 14. The design value of $C$ and $\sigma$ is 10 and 0.02, respectively. The number of support vector is 196 . Training and testing performance of MODEL II is $98.02 \%$ and $95.35 \%$, respectively. For SVM model, the performance of MODEL II is slightly better than the MODEL I. The reason is that the value of CSR creates lot of uncertainty in 


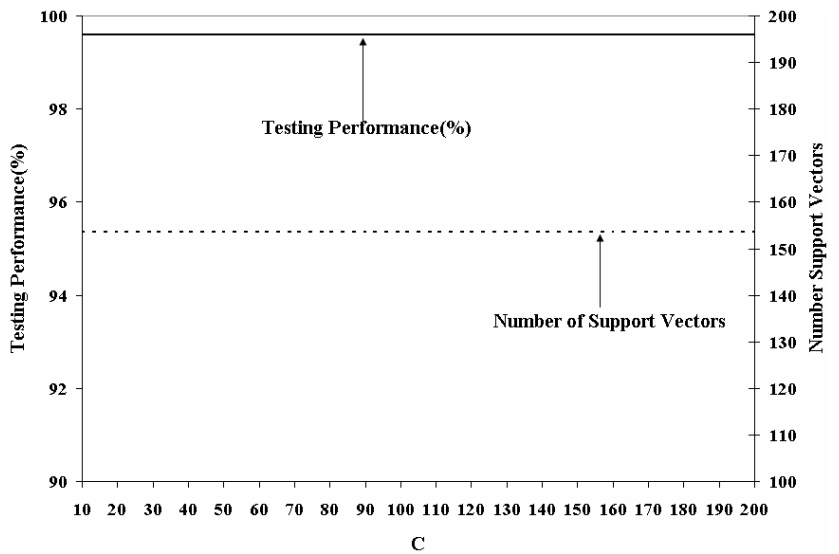

Fig. 13. Variation of Testing Performance (\%) and Number of Support Vectors with $\mathrm{C}$ values for MODEL II using radial basis function kernel.

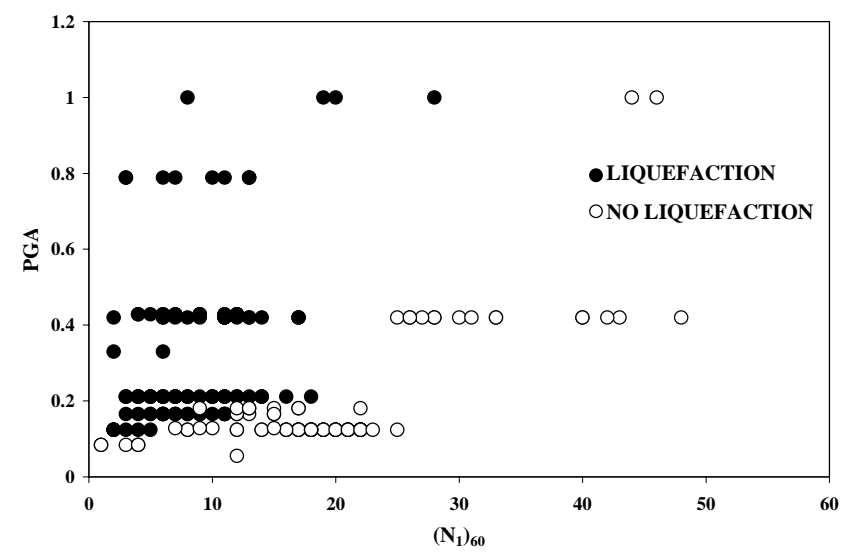

Fig. 14. Plot between PGA and $\left(N_{1}\right)_{60}$ for MODEL II using radial basis function for testing dataset.

MODEL I. The uncertainty arises from $r_{\mathrm{d}}$, unit weight, water table depth, etc. Figures 14 and 15 represent the plot between PGA and $\left(N_{1}\right)_{60}$ for training and testing dataset, respectively. The developed MODEL II has been verified for the different 85 case histories (which were not used either training or testing) available globally (Goh, 1994). In this case, the model performance is $74.12 \%$. So, SVM can be used as a practical tool for the prediction of liquefaction susceptibility of soil based on PGA and $\left(N_{1}\right)_{60}$. Figure 16 depicts the plot between PGA and $\left(N_{1}\right)_{60}$ for global data using SVM model.

A comparative study has been done between developed ANN and SVM model and it has been shown in Table 1 . For training dataset, the performance of ANN and SVM model is comparable. But for testing dataset and global data, SVM model outperforms ANN model. The use of the structural risk minimization principle in defining the cost function provided more generalization capacity with the SVM compared to the ANN, which uses the

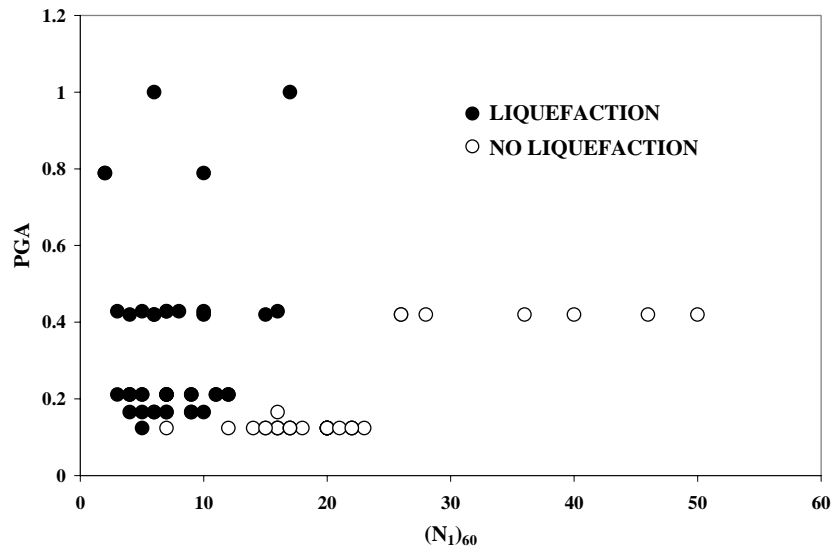

Fig. 15. Plot between PGA and $\left(N_{1}\right)_{60}$ for MODEL II using radial basis function for testing dataset.

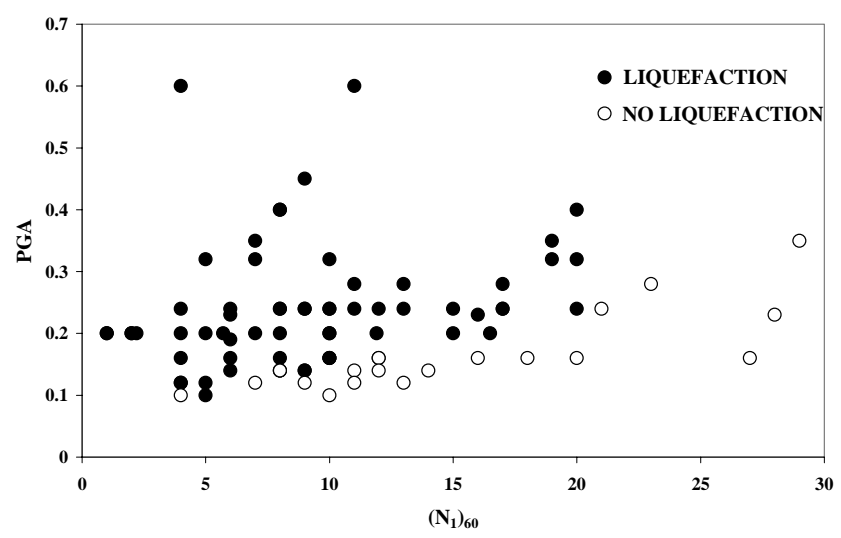

Fig. 16. Plot between PGA and $\left(N_{1}\right)_{60}$ for global data using radial basis function.

empirical risk minimization principle. SVM uses only two parameters $(\sigma$ and $C$ ). In ANN, there are a larger number of controlling parameters, including the number of hidden layers, number of hidden nodes, learning rate, momentum term, number of training epochs, transfer functions, and weight initialization methods. Obtaining an optimal combination of these parameters is a difficult task. Another advantage of the developed SVM is its optimization algorithm, which includes solving a linearly constrained quadratic programming function leading to a unique, optimal and global solution compared to the ANN. ANN model uses all training data for the final prediction. Whereas, SVM model employs only the support vector for final prediction. Therefore, the developed SVM produces a sparse solution. Sparseness means that a significant number of the weights are zero (or effectively zero), which has the consequence of producing compact, computationally efficient models, which in addition are simple and, therefore, produce smooth functions. This study 
Table 1. Comparison between ANN and SVM model.

\begin{tabular}{|c|c|c|c|c|c|c|}
\hline \multirow[t]{2}{*}{ Input variables } & ANN & SVM & ANN & SVM & \multirow{2}{*}{\multicolumn{2}{|c|}{$\begin{array}{c}\text { ANN SVM } \\
\text { [Global data] } \\
(\%)\end{array}$}} \\
\hline & \multicolumn{2}{|c|}{$\begin{array}{c}\text { [Training performance }] \\
(\%)\end{array}$} & \multicolumn{2}{|c|}{$\begin{array}{c}\text { [Testing performance] } \\
(\%)\end{array}$} & & \\
\hline $\mathrm{CSR},\left(N_{1}\right)_{60}$ & 94.55 & 96.04 & 88.37 & 94.19 & & \\
\hline PGA, $\left(N_{1}\right)_{60}$ & 94.05 & 98.02 & 87.20 & 95.35 & 70.58 & 77.65 \\
\hline
\end{tabular}

shows that SVM is a powerful computational tool to analyse the complex relationship between soil and seismic parameters in liquefaction analysis. As further field case records become available, the performance of the SVM can be improved. The developed SVM is simpler to apply than the method by Seed et al. (1971). Only minimal processing of the data are required, essentially to obtain values of $\left(N_{1}\right)_{60}$, for a given $a_{\max } / \mathrm{g}$.

\section{Conclusions}

ANN and SVM models have been developed for predicting liquefaction susceptibility of soil based on SPT data. For ANN model, the procedures to determine data division, data normalizing technique, network architecture selection, transfer function and the number of epochs are outlined. For SVM, The effect of $C$ on testing performance (\%) and the number of support vectors has been investigated. The MODEL II presented clearly that only two parameters [ $\left(N_{1}\right)_{60}$ and $\left.a_{\mathrm{max}} / \mathrm{g}\right]$ are sufficient input parameters for predicting liquefaction susceptibility of a site with depth. The performance of the developed models is encouraging for global dataset. The user can use the developed models (SVM and $\mathrm{ANN}$ ) as accurate and quick tools for the determination of liquefaction susceptibility of soil without any manual work such as using tables or charts. Comparison between SVM and ANN model indicates that SVM is a better model than ANN for predicting liquefaction susceptibility of soil based on SPT data.

Edited by: M. E. Contadakis

Reviewed by: two anonymous referees

\section{References}

Boser, B. E., Guyon, I. M., and Vapnik, V. N.: A training algorithm for optimal margin classifiers, in: 5th Annual ACM Workshop on COLT, 1 Pittsburgh, PA, edited by: Haussler, D., ACM Press, 44-152, 1992.

Cetin, K. O.: Reliability-based assessment of seismic soil liquefaction initiation hazard, Ph.D. dissertation, University of California, Berkeley, California, 2000.
Chu, B. L., Hsub, S. C., and Changa, Y. M.: Ground behavior and liquefaction analyses in central Taiwan-Wufeng, Eng. Geol., 71(1-2), 119-139, 2004.

Chua, D. B., Stewart, J. P., Leeb, S., Tsaic, J. S., Lind, P. S., Chud, B. L., Seede, R. B., Hsuf, S. C., Yug, M. S., and Wangh, M. C. H.: Documentation of soil conditions at liquefaction and nonliquefaction sites from 1999 Chi-Chi (Taiwan) earthquake, Soil Dyn. Earthq. Eng., 24(9-10), 647-657, 2004.

Cortes, C. and Vpanik, V.: Support-vector networks, Mach. Learn., 20(3), 273-297, 1995.

Demuth, H. B. and Beale, M.: Neural Network Toolbox, user's guide, MA: The Mathworks, Inc, Natick, 1999.

Fletcher, R.: Practical methods of optimization, 2nd edn., Wiley, Chichester, New York, 1987

Goh, A. T. C.: Seismic liquefaction potential assessed by neural networks, J. Geotech. Eng., 120(6), 1467-1480, 1994.

Gualtieri, J. A., Chettri, S. R., Cromp, R. F., and Johnson, L. F.: Support vector machine classifiers as applied to AVIRIS data, In the Summaries of the Eighth JPL Airbrone Earth Science Workshop, 1999.

Hagan, M. T. and Menhaj, M. B.: Training Feedforward Networks with the Marquardt Algorithm, IEEE T. Neural Networ., 5, 989993, 1994.

Juang, C. H., Haiming, Y., Lee, D. H., and Ku, C. S.: Assessing CPT-based methods for liquefaction with emphasis on the cases from the Chi-Chi, Taiwan, Earthquake, Soil Dyn. Earthq. Eng., 22, 241-258, 2002.

Kecman, V.: Learning And Soft Computing: Support Vector Machines, Neural Networks, And Fuzzy Logic Models, The MIT press, Cambridge, Massachusetts, London, England, 2001.

Keefer, D.: Landslides caused by earthquakes, Bull. Geol. Soc. Am., 95, 406-421, 1984.

Kramer, S. L.: Geotechnical Earthquake Engineering, Prentice Hall, New York, 1996.

Ku, C. S., Leeb, D. H., and Wuc, J. H.: Evaluation of soil liquefaction in the Chi-Chi, Taiwan earthquake using CPT, Soil Dyn. Earthq. Eng., 24(9-10), 659-673, 2004.

Kurup, P. U. and Dudani, N. K.: Neural networks for profiling stress history of clays from PCPT data, J. Geotech. Geoenviron., 128(4), 569-579, 2002.

Lee, C. T., Cheng, C. T., Liao, C. W., and Tsai, Y. B.: Site Classification of Taiwan Free-Field Strong-Motion Stations, B. Seismol. Soc. Am., 91(5), 1283-1297, 2001.

Lee, D. H., Juang, C. H., and Ku, C. S.: Liquefaction performance of soils at the site of a partially completed ground improvement project during the 1999 Chi-Chi earthquake in Taiwan, Can. Geotech. J., 38(6), 1241-1253, 2001. 
Liao, S. S. C., Veneziano, D., and Whitman, R. V.: Regression models for evaluating liquefaction probability, J. Geotech. Eng.ASCE, 114(3), 389-411, 1988.

Lin, A., Ouchib, T., Chenc, A., and Maruyamad, T.: Coseismic displacements, folding and shortening structures along the Chelungpu surface rupture zone occurred during the 1999 Chi-Chi (Taiwan) earthquake, Tectonophysics, 330(3-4), 225244, 2001.

More, J. J.: The Levenberg-Marquardt algorithm: Implementation and theory, Springer, Heidelberg, Numerical Analysis, edited by: Watson, G. A., 105-116, 1977.

Padmini, D., Ilamparuthi, K., and Sudheer, K. P.: Ultimate bearing capacity prediction of shallow foundations on cohesionless soils using neurofuzzy models, Comput. Geotech., 35, 33-46, 2008.

Papadopoulos, G. A. and Lefkopoulos, G.: Magnitude-distance relations for liquefaction in soil from earthquakes, B. Seismol. Soc. Am., 83(3), 925-938, 1993.

Park, D. and Rilett, L. R.: Forecasting freeway link travel times with a multi-layer feed forward neural network, Comput.-Aided Civ. Inf., 14358-14367, 1999.

Seed, H. B. and Idriss, I. M.: Analysis of soil liquefaction: Niigata earthquake, Journal of the Soil Mechanics and Foundation Divisions, ASCE, 93(3), 83-108, 1967.

Seed, H. B. and Idriss, I. M.: Simplified procedure for evaluating soil liquefaction potential, Journal of the Soil Mechanics and Foundation Divisions, ASCE, 97(6), 1249-1273, 1971.

Seed, H. B. and Idriss, I. M.: Ground Motions and Soil Liquefaction during Earthquakes, Earthquake Engineering Research Institute, Berkeley, California, 1982.

Seed, H. B., Idriss, I. M., and Arango, I.: Evaluation of liquefaction potential using field performance data, J. Goetech. Engrg. Div ASCE, 109(3), 458-482, 1983.

Seed, H. B., Tokimatsu, K., Harder, L. F., and Chung, R. M.: Influence of SPT procedures in soil liquefaction resistance evaluation, Rep. No. UCB/EERC-84/15, Earthquake Engrg. Res. Ctr., Univ. of California, Berkeley, Calif, 1984.
Shahin, M. A., Jaksa, M. B., and Maier, H. R.: Predicting the settlement of shallow foundations on cohesion less soils using back-propagation neural networks, Dept. of Civil and Envi. Eng., University of Adelaide, Australia, R167, 2000.

Shou, K. J. and Wang, C. F.: Analysis of the Chiufengershan landslide triggered by the 1999 Chi-Chi earthquake in Taiwan, Eng. Geol., 68(3-4), 237-250, 2003.

Sincero, A. P.: Predicting Mixing Power Using Artificial Neural Network, EWRI World Water and Environmental, 2003.

Sokolov, V. Yu, Loh, C. H., and Wen, K. L.: Comparison of the Taiwan Chi-Chi Earthquake Strong-Motion Data and GroundMotion Assessment Based on Spectral Model from Smaller Earthquakes in Taiwan, B. Seismol. Soc. Am., 92(5), 1855-1877, 2002.

Tang, Y., Zang, Y. Q., Huang, G., and Hu, X.: Granular SVMRFE Gene Selection Algorithm for Reliable Prostate Cancer Classification on Microarray Expression Data, Proceedings of the 5th IEEE Symposium on Bioinformatics and Bioengineering (BIBE'05), 2005.

Vapnik, V. N.: The nature of statistical learning theory, Springer, New York, 1995.

Vapnik, V. N.: Statistical learning theory, Wiley, New York, 1998.

Wang, C. Y., Dreger, D. S., Wang, C. H., Mayeri, D., and Berryman, J. G.: Field relations among coseismic ground motion, water level change and liquefaction for the 1999 Chi-Chi $\left(\mathrm{M}_{\mathrm{W}}=\right.$ 7.5) earthquake, Taiwan, Geophys. Res. Lett., 30(17), 1890, doi:10.1029/2003GL017601, 1890.

Wang, C. Y., Wang, C. H., and Manga, M.: Coseismic release of water from mountains: Evidence from the $1999(\mathrm{Mw}=7.5)$ ChiChi, Taiwan, earthquake, Geology, 32, 769-772, 2004

Wang, G. Q., Zhoua, X. Y., Zhangb, P. Z., and Igelc, H.: Characteristics of amplitude and duration for near fault strong ground motion from the 1999 Chi-Chi, Taiwan Earthquake, Soil Dyn. Earthq. Eng., 22(1), 73-96, 2002.

Yuan, H., Yang, S. H., Andrus, R. D., and Juang, C. H.: Liquefaction-induced ground failure: a study of the Chi-Chi earthquake cases, Eng. Geol., 71(1-2), 141-155, 2004. 